\title{
The Time Dynamics Research of Coherent State in Semiconductors by Excitation of Femtoseconds Pulses
}

\author{
V.N. TRUKhiN ${ }^{a}$ AND A.S. BUYsKIKH ${ }^{a, b}$ \\ ${ }^{a}$ A.F. Ioffe Physical Technical Institute, 194021 St. Petersburg, Russia \\ ${ }^{b}$ St. Petersburg State Polytechnic University, 194021 St. Petersburg, Russia
}

\begin{abstract}
In this article we present results of research of coherent processes of the ultrashort pulse light reemission in semiconductors. The time dynamics of the amplitude and the phase of the probe light wave reflected from the surface of low temperature GaAs was researched by the pump-probe method. The photodetector sensed the interference figure which shows interaction between probe pulse and pump pulse and also between probe pulse and excited by the pump coherent states in the semiconductor. The pulses wavelength is $800 \mathrm{~nm}$ and the pulses width is $15 \mathrm{fs}$. Thus the energy of excited photoelectrons was higher than conduction-band bottom by 60 to $140 \mathrm{meV}$. Due to the short duration of the pulse we could observe the coherent scattering of ultrashort light pulses at the room temperature. The impulse relaxation time for photoelectrons was determined by the experiments and it was $40 \mathrm{fs}$.
\end{abstract}

PACS: 06.60.Jn, 78.47.jh, 82.53.Kp, 78.45.+h

\section{Introduction and reasoning}

Nowadays there are several methods of researching the coherent fast light-induced processes in semiconductor media. For example, pump-probe experiments [1], by the means of this method the dynamics of changing of the reflection or the absorption of an excited sample could be researched. Also there are time-resolved correlation techniques with the femtoseconds time permission which allow to get a phase information $[2,3]$.

In the articles $[2,3]$ measurements and estimations of the exciton coherent state lifetime (dephasing time) in the medium was done by measuring of interference from optical pulses reflected from a semiconductor material. Through the reflection from the semiconductor surface the interferogram broadening was observed. This process is possible due to the exciton coherent states "lifetime" existence. It is significant that due to low temperatures it was possible to use long pulses (more than $100 \mathrm{fs}$ ): in this situation the dephasing time decreases due to the small mobility of charge carriers and their low interaction at these conditions. The dephasing time of researched GaAs was estimated and it was 1 ps.

Previous laws based on linear theory of the dispersion (low intensity) or the speed transport equations of radiation (the incoherent interaction) are not exactly correct at expansion of ultrashort pulses through the resonance absorbed medium.

The common property of the new effects are relaxation processes (collisions, spontaneous emission and etc.) which do not have enough time to destroy the "phase memory" of excited states. Due to this fact, the medium polarization is non-linear function of amplitude and field phase in the all previous time periods. The striking example of the "phase memory" is the self-induced transparency [4]. At intensity higher than critical significance the pulse absorption decreases quickly and the previously absorbed substance becomes almost transparent for radiation. This process is accompanied by the significant decrease of the pulse velocity in comparison with the phase speed in the medium.

The physics of this phenomenon can be explained by examination of dynamics interacting between the pulse and the medium. The optically coupled electron-hole states become excited during the passage of the pulse front edge through the medium. Consequently, the part of field energy remains in the medium. If the pulse intensity is high enough (1), the stimulated radiation becomes overridden by the spontaneous radiation

$$
\frac{\mu}{\hbar} \int_{-\infty}^{+\infty} E(y, t) \mathrm{d} t>\pi
$$

$\mu$ is the absolute value of the non-diagonal element of the dipole atom moment, $E(y, t)$ is the electromagnetic field. The excited medium induces radiation during the passage of the residual part of the pulse, thereby the energy returns to the back front of pulse. Due to the fact that the pulse duration is lower than the relaxation time, this process is quick enough to not let the relax process destroy the coherence of interaction. So, there is a possibility to make the situation where all energy, which was taken from the field previously returns to the pulse, recovering the original waveform. So, the pulse can move through the absorbed medium without losses, giving back and 
taking up its energy constantly. That is why the moving speed decreases. Here is necessary to divide the coherent bleaching of the medium and the bleaching under the usual absorption saturation, which takes place when the powerful pulse duration is longer than the dephasing time in the medium. In the last case relaxation processes are important in interaction: medium absorption or emission of the energy becomes simply proportional to the difference between electron and hole state populations. So, this pulse cannot transfer the medium into the inverse state and the biggest effect is equalization of the probabilities of occupancies of coupled electron-hole states. The energy, which is necessary for the process, is taken from the pulse and cannot be returned by the coherent way. The energy transfers to the energy of the spontaneous radiation or non-radiative transitions, but it is lost for the pulse. So, the energy for the medium blooming is taken from the pulse energy irreversibly.

\section{Experimental considerations}

In this article experiments devoted to the optical coherent transition in the semiconductor medium were done by the time-resolved correlation techniques. Two ultrashort pulses collinearly irradiate the semiconductor surface. One of the pulses has low power, the optical probe pulse, and the second pulse is the optical pump pulse, and its power could be changed from about probe values to higher values. The physics of the process is the same as in the earlier described self-induced transparency. There is a difference between these processes: the stimulated radiation now depends on both pulses. In case of the low pump we do not observe the stimulated radiation because it is less than the spontaneous radiation. In case of the high pump the high level population exceeds the low level population for the optical coupled states. During the passage of the probe pulse through the excited medium, the last one produces the stimulation radiation with the same phase as the pump wave and the power of probe pulse rises. In case of the high carrier density the "lifetime" of coherent state is generally defined by the time of electron-electron collisions that is the impulse relaxation time of carriers.

\section{Results}

The experimental apparatus is analogous to that used for time-resolved luminescence correlations [5]. The measurements were made at the room temperature. We used femtoseconds Ti:sapphire laser with mean wavelength $800 \mathrm{~nm}, 15$ fs pulse duration, $80 \mathrm{MHz}$ repetition rate. The laser beam was divided by beamsplitter: the first beam (pump pulse) went through mobile delay line and another beam (probe pulse) went through stationary delay line. Then, the collinear beams were focused with the same polarization, onto the Brewster-angled sample and further they were directed into a photodetector. The angle of incidence is set up to the Brewster angle to increase the transmission of the wave inside of semiconductor slab. The probe pulse was modulated and the signal corresponding to the electromagnetic radiation at the modulation frequency was registered by the lock-in amplifier. The frequency of modulating was $1190 \mathrm{~Hz}$. The coherent transient was probed by the time integrated detection of the signal as a function of the delay $\tau_{\mathrm{D}}$.

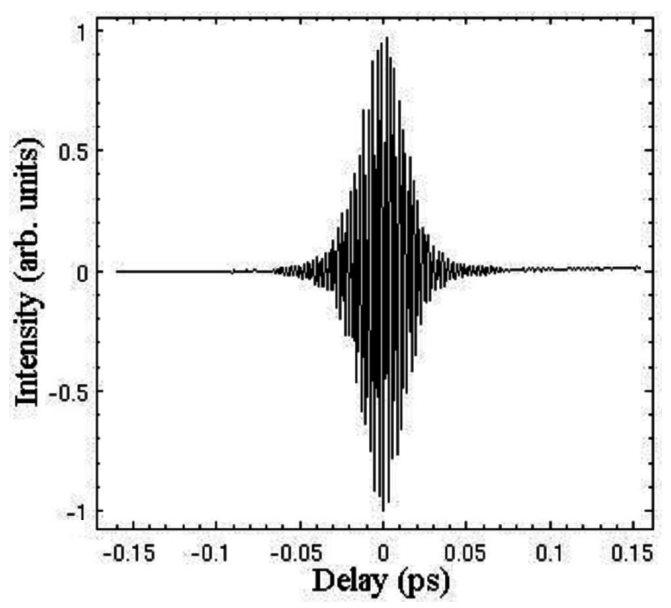

Fig. 1. The interference pattern on conditions that pulses reflected from slab with $\mathrm{Au}$ deposition.

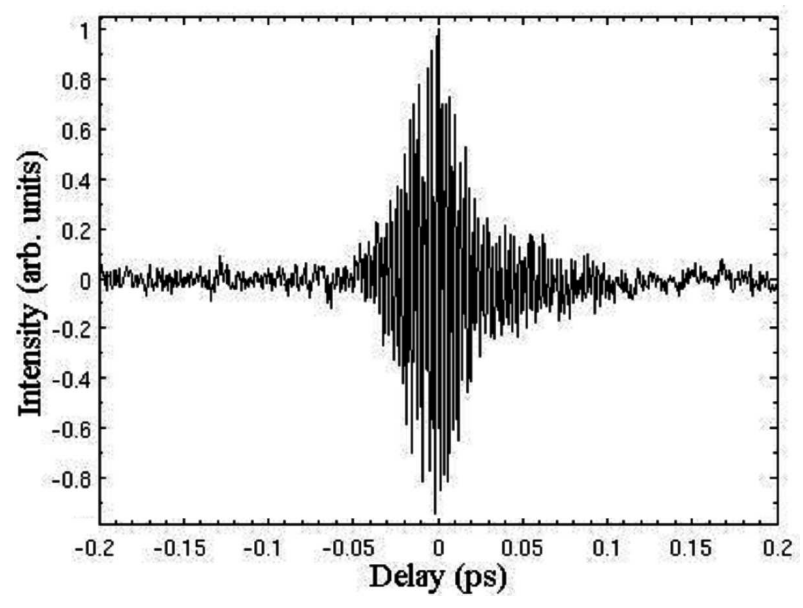

Fig. 2. The interference pattern on conditions that pulses reflected from LT-GaAs. $I_{\text {pump }} \approx 100 \mathrm{MW} / \mathrm{cm}^{2}$.

Primarily we obtained the interference pattern on conditions that pulses reflected from slab with $200 \mathrm{~nm} \mathrm{Au}$ deposition. As a result we obtained pulses' forms (Fig. 1). For investigation of the coherent luminescence from the semiconductor medium we used low temperature (LT)-GaAs slab. The interferogram is symmetric in case of the low pump, it looks like in Fig. 1.

The interferogram is asymmetric in case of the high level excitation in the direction of the pump pulse lead (Fig. 2). It indicates that the medium transfers the en- 
ergy from the pump pulse to the probe pulse. The impulse relaxation time of charge carriers was estimated for the exponential decay of the coherent polarization relaxation $\approx 40 \mathrm{fs}$

\section{Conclusion}

By researching the "lifetime" of the coherent states in semiconductor medium we made the direct measurements of the impulse relaxation time of charge carriers. The numerical values obtained well conform to results of the early works in which the impulse relaxation time of charge carriers was obtained by the time dynamics research of the beam transmission through thin GaAs slab.

\section{References}

[1] C.V. Shank, in: Ultrashort Laser Pulses and Application, Eds. W. Kaiser, D.H. Auston, Springer-Verlag, Berlin 1988, p. 5

[2] S. Ceccherini, F. Bogani, M. Gurioli, M. Colocci, Opt. Commun. 132, 77 (1996).

[3] M. Gurioli, F. Bogani, S. Ceccherini, M. Colocci, Phys. Rev. Lett. 78, 3205 (1997).

[4] I.A. Poluektov, Yu.M. Popov, JETP Lett. 9, 542 (1969).

[5] R. Strobel, R. Eccleston, J. Kuhl, K. Köhler, Phys. Rev. B 43, 12564 (1991). 